\title{
Reproducibility, temporal stability, and functional correlation of diffusion MR measurements within the spinal cord in patients with asymptomatic cervical stenosis or cervical myelopathy
}

\author{
Benjamin M. Ellingson, PhD, ${ }^{1-4}$ Noriko Salamon, MD, ${ }^{1}$ Davis C. Woodworth, BS,, ${ }^{1,2}$ \\ Hajime Yokota, MD, ${ }^{1}$ and Langston T. Holly, MD ${ }^{5}$
}

Departments of ${ }^{1}$ Radiological Sciences, ${ }^{2}$ Biomedical Physics, ${ }^{4}$ Psychiatry and Biobehavioral Sciences, and ${ }^{5}$ Neurosurgery and Orthopaedics, David Geffen School of Medicine; and ${ }^{3}$ Department of Bioengineering, Henri Samueli School of Engineering and Applied Sciences, University of California, Los Angeles, California

OBJECTIVE The purpose of this study was to quantify the reproducibility, temporal stability, and functional correlation of diffusion MR characteristics in the spinal cord in patients with cervical stenosis with or without myelopathy. The association between longitudinal diffusion tensor imaging (DTI) measurements and serial neurological function assessment was explored at both the group and individual level.

METHODS Sixty-six nonoperatively treated patients with cervical stenosis were prospectively followed (3 months to > 5 years) using synchronous serial MRI and functional outcome assessment. A total of 183 separate MRI examinations were performed, separated by at least 3 months, and each patient had a minimum of 2 MRI scans (range 2-5 scans). Anatomical and DTI measurements were performed within the spinal cord at the C1-2 region as well as at the area of highest compression. Coefficients of variance (COVs) were compared across measurements in both reference tissue and areas of compression for anatomical measurements, fractional anisotropy (FA), and mean diffusivity (MD). The correlation between diffusion MR measures at the site of compression and evaluations of neurological function assessed using the modified Japanese Orthopaedic Association (mJOA) scale at multiple time points was evaluated.

RESULTS The COVs for anatomical measurements (Torg ratio and canal diameter) were between $7 \%$ and $10 \%$. The median COV for FA measurements at the site of compression was 9\%, and for reference tissue at C1-2 it was 6\%. The median COV for MD at the site of compression was approximately $12 \%$, and for reference tissue at C1-2 it was $10 \%$. The FA and MD measurements of $\mathrm{C} 1-2$ averaged 0.61 and $0.91 \mu \mathrm{m}^{2} / \mathrm{msec}$, respectively, whereas the FA and MD measurements at the site of compression averaged 0.51 and $1.26 \mu \mathrm{m}^{2} / \mathrm{msec}$, respectively. Both $F A$ (slope $=0.037 ; R^{2}=$ $0.3281, p<0.0001$ ) and MD (slope $=-0.074 ; R^{2}=0.1101, p=0.0084$ ) were significantly correlated with the mJOA score. The FA decreased by approximately 0.032 units per mJOA unit decrease $\left(R^{2}=0.2037, p<0.0001\right)$, whereas the MD was increased by approximately $0.084 \mu \mathrm{m}^{2} / \mathrm{msec}$ for every mJOA unit decrease $\left(R^{2}=0.1016, p<0.0001\right)$.

CONCLUSIONS Quantitative DTI measurements of the spinal cord in patients with cervical stenosis with or without myelopathy have a median COV of $5 \%-10 \%$, similar to anatomical measurements. The reproducibility of these measurements and significant correlation with functional outcome status suggest a potential role in the evaluation and longitudinal surveillance of nonoperatively treated patients. With respect to the specific DTI measurements, FA within the spinal cord appears slightly more sensitive to neurological function and more stable than measures of MD. Therefore, DTI of the spinal cord may be a clinically feasible imaging technique for longitudinally monitoring patients with cervical spondylotic myelopathy.

https://thejns.org/doi/abs/10.3171/2017.7.SPINE176

KEY WORDS diffusion tensor imaging; DTI; spinal cord; cervical spondylotic myelopathy; biomarker; CSM; stenosis

ABBREVIATIONS COV = coefficient of variance; $C S M=$ cervical spondylotic myelopathy; DTI = diffusion tensor imaging; FA = fractional anisotropy; FOV = field of view; $\mathrm{MD}=$ mean diffusivity; $\mathrm{mJOA}=$ modified Japanese Orthopaedic Association

SUBMITTED January 5, 2017. ACCEPTED July 28, 2017.

INCLUDE WHEN CITING Published online February 9, 2018; DOI: 10.3171/2017.7.SPINE176. 


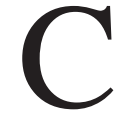
ERVICAL spondylosis results from degeneration of intervertebral discs and supporting structures, which collapse and dehydrate during normal aging, ${ }^{2}$ increasing mechanical stress at cartilaginous endplates at the edge of the vertebral bodies. ${ }^{3,15,25,32}$ Over time, this repeated stress results in subperiosteal bone formation and/or osteophyte formation. Symptoms then arise during repeated spinal cord or nerve root compression ${ }^{2,5,8}$ and manifest as neck pain syndromes, myelopathy, or radiculopathy. $1,5,11$

Cervical spondylosis is diagnosed using anatomical MRI and is ubiquitous in the elderly. Myelopathy resulting from spondylosis is the most common cause of spinal cord dysfunction in the elderly; ${ }^{47}$ however, accurate estimation of the dysfunction attained using standard MRI remains a significant challenge, because common anatomical features, ${ }^{4,17}$ including MR signal change ${ }^{16,28-31,33,34,37}$ and the degree of spinal cord compression, ${ }^{36,42,48}$ have not demonstrated a reliably strong and/or consistent association with neurological function. Decompression surgery is commonly performed in patients with moderate and severe myleopathy, whereas patients with asymptomatic stenosis or mild symptomatology may be treated nonoperatively and observed, without continual progression ${ }^{8,26}$, 27,35 in many cases. Due to the aforementioned limitations of conventional MRI, there is significant interest in the development of noninvasive imaging biomarkers to quantify neurological function of the spinal cord in both operatively and nonoperatively treated patients with advanced cervical spondylosis.

Diffusion tensor imaging (DTI), an advanced MR technique sensitive to the underlying microstructural organization of tissues,${ }^{41}$ has previously been shown to be useful for predicting neurological function in patients with cervical spondylosis. ${ }^{6,10,21,23,24,44,45}$ In particular, studies have shown that lowered diffusion fractional anisotropy (FA) at the site of compression is associated with increased neurological dysfunction, suggesting disruption in the directional coherence of nerve fibers in the spinal cord, and higher mean diffusivity (MD) of water at the site of compression is associated with increasing neurological impairment.

Most DTI studies in patients with spondylosis have been cross-sectional, examining the relationship between DTI and functional status at a single time point; the variability and longitudinal stability of DTI measurements in the spinal cord in advanced cervical spondylosis have not been previously reported. The current study quantified the variation in DTI measurements by examining repeated MRI investigations in a cohort of patients with asymptomatic cervical stenosis and cervical spondylotic myelopathy (CSM) who were treated nonoperatively and prospectively monitored. The association between longitudinal DTI measurements and serial neurological function assessment was explored at both the group and individual level.

\section{Methods}

\section{Patient Population}

A prospective observational study was performed in 66 nonoperatively treated patients with cervical stenosis with or without myelopathy who were initially referred for surgical evaluation. Each patient had 2 or more DTI and conventional MRI scans, and a total of 183 separate MRI scans were performed across the entire cohort (mean 2.7 scans per patient, range $2-5$ separate scans). The total surveillance time ranged from 3 months to more than 5 years (average follow-up duration per patient -520 days). The patients underwent synchronous MRI and neurological assessment at the same uniform time points: baseline, 3 months, 6 months, 1 year, 2 years, 3 years, and 5 years following their initial evaluation. Thirty-seven of the patients were male and 29 were female, and the average age was 64 years (range 24-94 years).

The degree of neurological impairment was measured using the modified Japanese Orthopaedic Association $(\mathrm{mJOA})^{46}$ scale at the same time as each MRI scan. Fortyeight patients had some evidence of neurological symptomatology, as indicated by an mJOA score of $<18$, and 18 patients had no neurological symptomatology but reported neck pain. The average mJOA score was 16.6 , with a range from 11 to 18. All participants gave informed written consent to be part of this study. Surgical management was discussed with any patient whose mJOA score declined by 2 or more points during the observation period. However, none of these patients opted for surgical intervention. All procedures complied with the principles of the Declaration of Helsinki and were approved by the Institutional Review Board within the Office for the Protection of Research Subjects at the University of California.

\section{Conventional MRI}

Standard MRI was obtained on a 3-T MR scanner (3T Trio or Prisma; Siemens Healthcare) using a standard spine coil array for radiofrequency reception. Routine clinical MRI scans consisted of T1-weighted and T2-weighted sequences in the sagittal plane and T2-weighted images in the axial orientation. All patients had radiographic evidence of at least moderate cervical stenosis-including spinal canal narrowing related to advanced cervical spondylosis - manifested by a combination of facet arthropathy, ligamentum flavum hypertrophy, and varying degrees of ventral disc-osteophyte compression. An MRI version of the Torg-Pavlov ratio ${ }^{36}$ (ratio of anterior-posterior diameter to thickness of the vertebral body) was documented and used for subsequent comparisons with diffusion measurements.

\section{Diffusion Tensor Imaging}

Axial diffusion-weighted images were collected through the level of most significant canal narrowing. Excitation consisted of a custom 2D, spatially selective, radiofrequency excitation pulse and a reduced field of view (FOV) echo planar imaging readout with ramp sampling (Zoomed-EPI). The TE was set to 73-100 msec and the TR to $3000-10,000$ msec; matrix size $48 \times 128$; FOV $53 \mathrm{~mm} \times 140 \mathrm{~mm}$; slice thickness $4-5 \mathrm{~mm}$ with no gap; number of averages 4-10; and there were 12-20 diffusionsensitizing directions with $b=500$ seconds $/ \mathrm{mm}^{2}$ and $1-2$ $\mathrm{b}=0$ seconds $/ \mathrm{mm}^{2}$ images. After acquisition of diffusionweighted images, eddy-current and motion correction was 


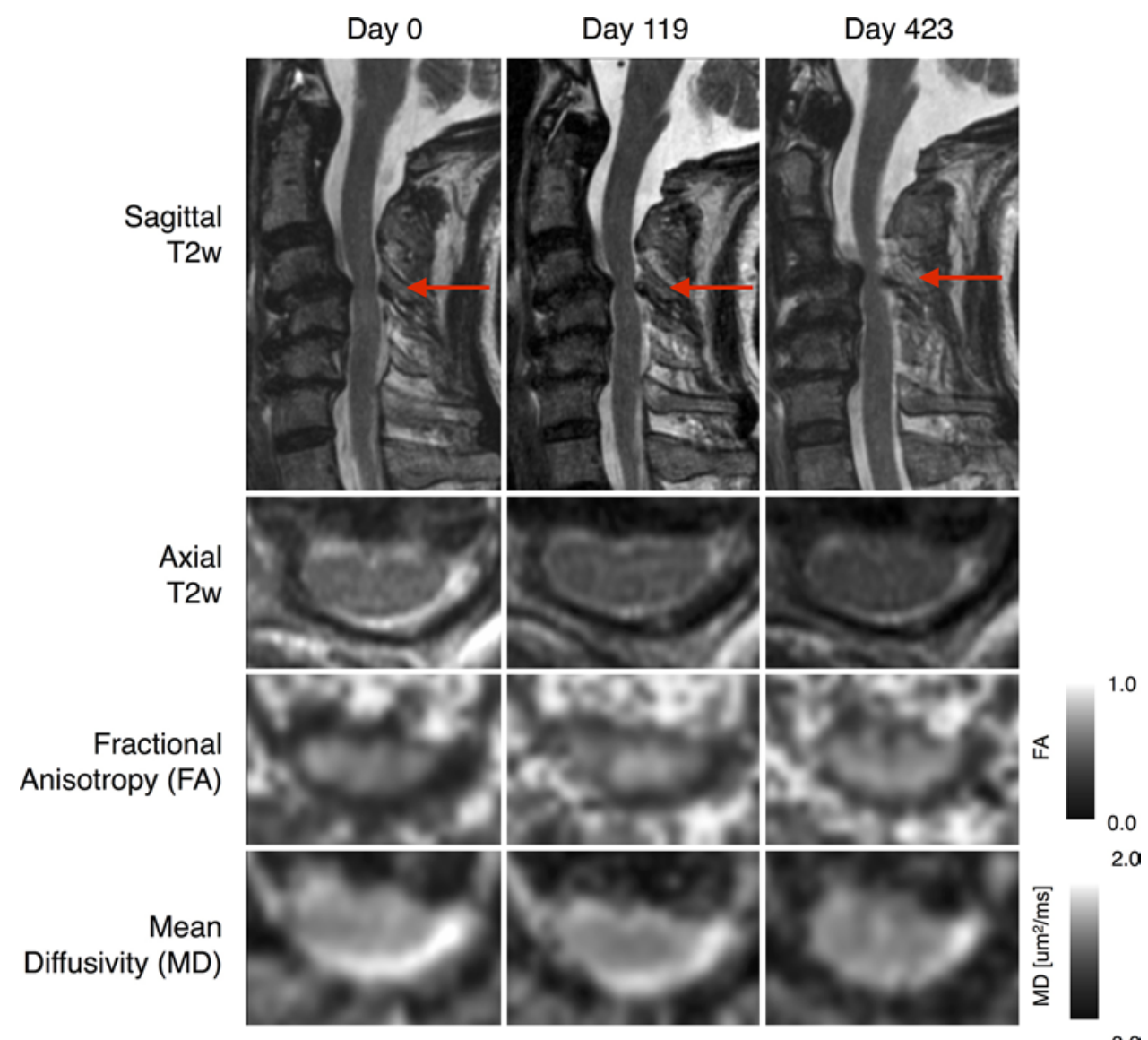

FIG. 1. Serial longitudinal anatomical and DTI measurements obtained in a 77-year-old woman with spinal cord compression at C3-4 and subtle T2 signal change, but no neurological impairment (mJOA score 18). Red arrows show the site of most significant cervical stenosis and area of evaluation. Figure is available in color online only.

performed using a 12-degrees-of-freedom affine transformation included in the FSL software (FMRIB; Oxford, UK; http://www.fmrib.ox.ac.uk/fsl/). The MD, or average apparent diffusion coefficient relating to the mean water motility, and FA, the degree of diffusion anisotropy, were calculated from the resulting diffusion MR data.

\section{Regions of Interest}

Manual segmentation of the spinal cord was performed for the whole cord at each axial image slice location by using the T2-weighted anatomical images. The DTI measurements at the site of highest compression, including voxels in areas of T2 hyperintensity, were used for comparison. The DTI measurements of the cord at the C1-2 spinal levels were also made for a reference.

\section{Statistical Analysis}

The coefficient of variance (COV) was calculated by estimating the ratio of the standard deviation of the mean FA and MD in the spinal cord over time, divided by the average of the mean FA and MD measurements over the same time period $(\mathrm{COV}=\sigma / \mu)$. Temporal trends in both average and percentage change in FA and MD were displayed for each individual patient. Last, the linear relationship between DTI measurements and mJOA scores was determined using Pearson's correlation coefficient and multiple measurements per patient. For these associations, statistical significance was determined by testing whether the slope of the linear trend line was significantly different from zero by using an F-test. All statistical analyses were performed using GraphPad Prism v6.0d (GraphPad Software, Inc.) and MATLAB (R2011b; MathWorks, Inc.).

\section{Results}

\section{The COV in Repeated Anatomical and DTI Measurements of the Spine}

Figure 1 provides example images from a neurologically stable patient with cervical stenosis, demonstrating the relative consistency in DTI measurements over time. The median mJOA score for the patient cohort was 17 , the median FA at the lesion site was 0.50 , the median MD at the lesion site was $1.25 \mu \mathrm{m}^{2} / \mathrm{msec}$, and the median MRI-equivalent Torg ratio was 0.36 . The median COV was significantly different across anatomical and DTI measurements (Friedman test, $\mathrm{p}<0.0001$ ), indicating that COVs in repeated DTI measurements were slightly higher than anatomical measurements of the spinal column, and there was substantial variability when examining repeated MD measurements at the site of compression. More pre- 

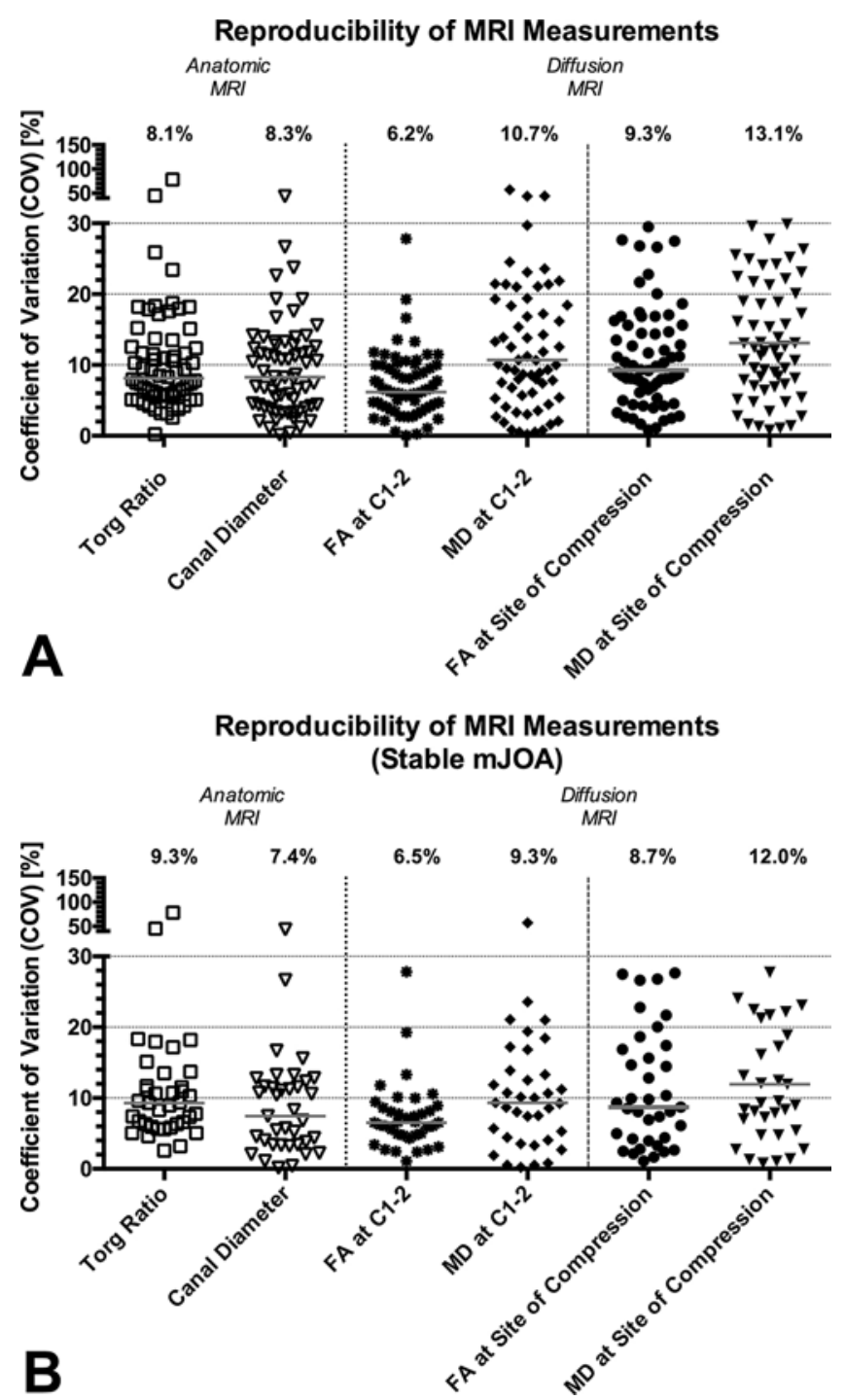

FIG. 2. Graphs showing the COV in repeated anatomical and DTI measurements of the spinal cord in patients with cervical spondylosis. A: The COV in all patients $(\mathrm{N}=66)$. B: The COV in only patients with no change in mJOA score $(n=37)$.

cisely, the median COV for anatomical measurements of the MRI-equivalent Torg ratio was $8.1 \%$ and the median COV for measurements of canal diameter was $8.3 \%$ (Fig. $2 \mathrm{~A}$, Table 1), whereas the median COVs of FA and MD measurements at the site of compression were $9.3 \%$ and $13.1 \%$, respectively. Dunn's test for multiple comparisons indicated that both canal diameter (adjusted $p=0.0012$ ) and Torg ratio (adjusted $\mathrm{p}=0.0073$ ) had a significantly lower median COV compared with the median COV of $\mathrm{MD}$ at the site of compression, but no difference in COV was detected for canal diameter or Torg ratio when these values were compared with FA (adjusted $p>0.05$ ). For reference, the median COVs of FA and MD measurements in reference tissue at spinal level C1-2 were $6.2 \%$ and $10.7 \%$, respectively, and the median COV for FA was significantly lower than that for MD in these reference tissues (adjusted $\mathrm{p}=0.0121$ ). Additionally, there was a significant difference in the median FOV between FA measurements at $\mathrm{Cl}-2$ (lowest COV) and MD at the site of compression (highest COV) (adjusted $\mathrm{p}<0.0001$ ). When examining COVs in patients who had no change in mJOA score, trends were similar (Fig. 2B, Table 2; $\mathrm{p}=0.5855$ comparing median COV for all patients vs median COV for the subset with constant mJOA scores).

\section{Temporal Patterns in DTI Measurements of the Spinal Cord in Cervical Spondylosis During Long-Term Surveillance}

Multiple independent DTI measurements (mean \pm SD) of reference spinal cord tissue at $\mathrm{C} 1-2$ for all patients resulted in an average FA measurement of $0.61 \pm 0.06$ and an MD measurement of $0.91 \pm 0.20 \mu \mathrm{m}^{2} / \mathrm{msec}$ (Fig. 3A and B). Grouping all patients with CSM together and assuming that each DTI scan represents an independent measurement, we found the average FA at the site of compression to be $0.51 \pm 0.10$ and the average MD to be $1.26 \pm$ $0.35 \mathrm{\mu m}^{2} / \mathrm{msec}$, which was significantly lower than those of the reference tissues (paired t-test, $\mathrm{p}<0.0001$ for both FA and MD). Measurements both at $\mathrm{C} 1-2$ and at the site of compression did not show linear or polynomial trends $(p>$ 0.1 ), suggesting stability of these measurements over time.

Longitudinal DTI measurements for both FA and MD at the site of compression for individual patients during the period of radiographic surveillance are illustrated in Fig. 3C and D. The percentage changes in DTI measurements for FA and MD from the first evaluation time point are illustrated in Fig. 3E and F. Despite some fluctuations in DTI acquisition protocols over the period of evaluation, the majority of the patients (47 of 66) showed relatively steady measurements over time, consistent with their stable neurological status. However, a few patients (13 of 66) did demonstrate fluctuations (> 10\% COV) in DTI measurements over time. In some of these cases ( 7 of 13), significant issues with DT image quality, including severe susceptibility artifacts, were observed only at specific time points. In 2 of 13 patients, these artifacts were present in $>2$ evaluation time points. In the remaining 4 patients, spinal cord motion or other issues may have contributed to the high fluctuations in measurements.

\section{Functional Correlation of DTI Measurements}

Next, we examined the relationship between DTI measurements and mJOA scores to determine whether FA or MD were valuable predictors of neurological status. Pooling all DTI measurements from all patients and all time points, both FA and MD at the site of compression showed a significant correlation with neurological status (Fig. 4A and B). Specifically, FA was shown to decrease approximately 0.032 units per mJOA unit decrease $\left(R^{2}=0.2037\right.$, $\mathrm{p}<0.0001$, whereas MD was shown to increase approximately $0.084 \mu \mathrm{m}^{2} / \mathrm{msec}$ for every mJOA unit decrease $\left(\mathrm{R}^{2}\right.$ $=0.1016, \mathrm{p}<0.0001)$. When evaluating each patient individually by averaging both their DTI and mJOA findings over their respective surveillance period, $\mathrm{FA}\left(\mathrm{R}^{2}=0.3281\right.$, $\mathrm{p}<0.0001)$ and $\mathrm{MD}\left(\mathrm{R}^{2}=0.1101, \mathrm{p}=0.0084\right)$ were correlated with approximately the same sensitivity to the mJOA score (Fig. 4C and D). Together, these results further support the hypothesis that DTI measurements at the site of 
TABLE 1. The COV for anatomical and diffusion MRI measurements in all 66 patients in the cohort

\begin{tabular}{lrrrccr}
\hline \multicolumn{1}{c}{ Measurement } & $\begin{array}{c}\text { Canal } \\
\text { Diameter }\end{array}$ & $\begin{array}{c}\text { Torg } \\
\text { Ratio }\end{array}$ & $\begin{array}{c}\text { FA at } \\
\text { C1-2 }(\%)\end{array}$ & $\begin{array}{c}\text { MD at } \\
\text { C1-2 (\%) }\end{array}$ & $\begin{array}{c}\text { FA at Site of } \\
\text { Compression (\%) }\end{array}$ & $\begin{array}{c}\text { MD at Site of } \\
\text { Compression (\%) }\end{array}$ \\
\hline Minimum & 0.1 & 0.2 & 0.0 & 0.2 & 0.8 & 0.8 \\
\hline 25th percentile & 4.2 & 5.6 & 4.0 & 5.6 & 5.9 & 7.8 \\
\hline Median & 8.3 & 8.1 & 6.2 & 10.7 & 9.3 & 13.1 \\
\hline 75th percentile & 12.8 & 12.8 & 10.0 & 19.8 & 15.6 & 23.4 \\
\hline Maximum & 44.0 & 78.4 & 27.8 & 57.1 & 29.5 & 34.6 \\
\hline Mean & 9.6 & 11.1 & 7.1 & 13.8 & 11.0 & 15.3 \\
\hline SD & 7.2 & 10.9 & 4.6 & 11.4 & 7.1 & 9.7 \\
\hline SEM & 0.9 & 1.3 & 0.6 & 1.4 & 0.9 & 1.2 \\
\hline Lower $95 \%$ Cl of mean & 7.8 & 8.4 & 6.0 & 11.0 & 9.3 & 12.9 \\
\hline Upper $95 \%$ Cl of mean & 11.4 & 13.8 & 8.3 & 16.6 & 12.8 & 17.7 \\
\hline
\end{tabular}

$\mathrm{SEM}=$ standard error of the mean.

compression, particularly FA and $\mathrm{MD}$, may be valuable predictors of neurological status.

\section{Discussion}

Degenerative changes within the cervical spine are a normal part of aging and are commonly encountered in middle-aged and older patients. Because of this ubiquity among older patients, one of the greatest challenges associated with the clinical management of patients with advanced cervical spondylosis is determining when to intervene, either surgically or therapeutically, because many changes within the spinal cord may be relatively benign and may not result in permanent neurological damage. Additionally, patients may have similar-appearing conventional MRI features, such as degree of stenosis and spinal cord signal change, yet have distinctly different degrees of functional impairment. Moreover, because patients with asymptomatic and mildly symptomatic CSM are frequently treated nonoperatively, a tool for obtaining objective measurements of spinal cord integrity would be highly beneficial for long-term surveillance and clinical management of this patient population.

Although conventional MRI is the current standard for radiographic assessment of the spinal cord in patients with CSM, measures including spinal cord compression, ${ }^{36,42,48}$ spinal canal diameter, and even the presence of spinal cord signal change $e^{16,28-31,33,34,37}$ have failed to show a consistent, significant association with neurological impairment in these patients. To overcome these limitations, studies have explored the use of advanced imaging techniques, such as DTI, $, 9,10,19-21,23,28,38,44$ to inspect microstructural characteristics and to evaluate the degree of spinal cord injury and functional impairment. Despite promising initial studies, questions remain regarding the reproducibility and reliability of using DTI as a tool for longitudinal surveillance of the spinal cord in patients with spinal cord disease.

To address this concern, in the current study we sought to investigate the reproducibility, temporal trends, and functional correlates of DTI measurements within the cervical spinal cord in a large cohort of patients with asymptomatic cervical stenosis or CSM over a surveillance period that ranged from 3 months to more than 5 years. We report a COV for repeated measurements in patients with cervical spondylosis of between $5 \%$ and $10 \%$, similar to the variability in simple anatomical measurements and similar to the variability in DTI parameters reported in the literature. A previous study by our team showed COVs

TABLE 2. The COV for anatomical and diffusion MRI measurements in 37 patients with no change in mJOA

\begin{tabular}{lcccccc}
\hline \multicolumn{1}{c}{ Measurement } & $\begin{array}{c}\text { Canal } \\
\text { Diameter }\end{array}$ & $\begin{array}{c}\text { Torg } \\
\text { Ratio }\end{array}$ & $\begin{array}{c}\text { FA at } \\
\text { C1-2 }(\%)\end{array}$ & $\begin{array}{c}\text { MD at } \\
\text { C1-2 (\%) }\end{array}$ & $\begin{array}{c}\text { FA at Site of } \\
\text { Compression (\%) }\end{array}$ & $\begin{array}{c}\text { MD at Site of } \\
\text { Compression (\%) }\end{array}$ \\
\hline Minimum & 0.1 & 2.6 & 1.1 & 1.1 & 0.8 & 0.2 \\
\hline 25th percentile & 3.4 & 6.1 & 4.1 & 4.7 & 6.2 & 4.2 \\
\hline Median & 7.4 & 9.3 & 6.5 & 9.3 & 8.7 & 12.0 \\
\hline 75th percentile & 12.0 & 12.6 & 17.1 & 8.8 & 22.3 & 15.4 \\
\hline Maximum & 44.0 & 78.4 & 27.7 & 27.8 & 34.1 & 57.1 \\
\hline Mean & 9.0 & 12.2 & 11.2 & 7.4 & 14.2 & 11.4 \\
\hline SD & 8.1 & 13.3 & 8.2 & 4.9 & 10.2 & 10.6 \\
\hline SEM & 1.3 & 2.2 & 1.4 & 0.8 & 1.7 & 1.7 \\
\hline Lower $95 \%$ Cl of mean & 6.3 & 7.8 & 8.4 & 5.8 & 10.8 & 7.9 \\
\hline Upper $95 \%$ Cl of mean & 11.7 & 16.7 & 13.9 & 9.0 & 17.6 & 15.0 \\
\hline
\end{tabular}


Fractional Anisotropy (FA) vs. Time at Site of Compression \& C1-2 Reference

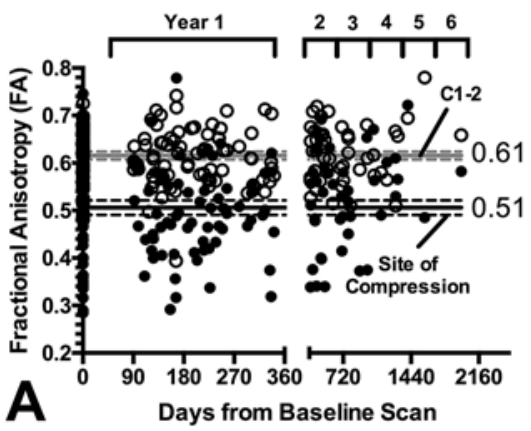

Fractional Anisotropy (FA) vs. Time for Individual Patients at Site of Compression

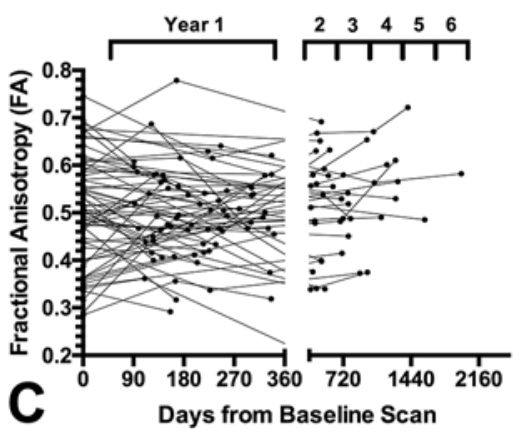

Percentage Change in Fractional Anisotropy (FA) vs. Time for Individual Patients at Site of Compression

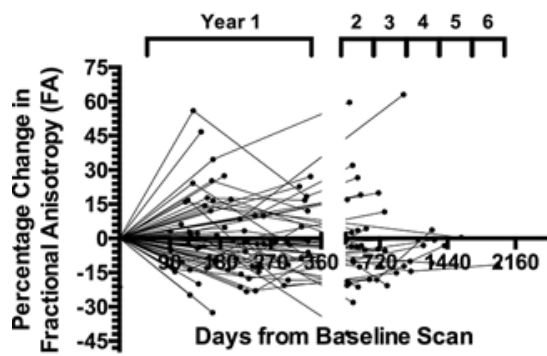

E

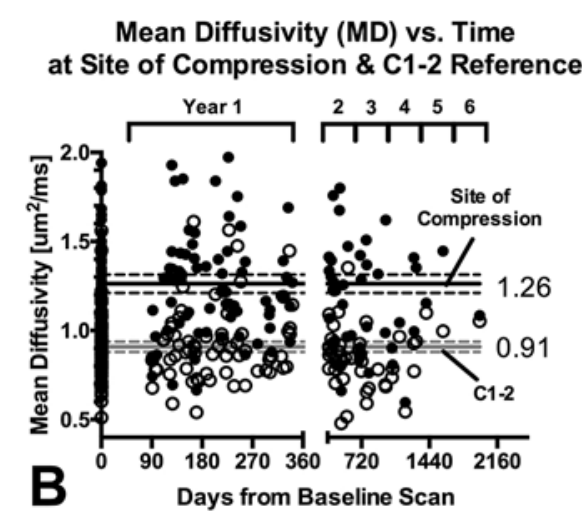

Mean Diffusivity (MD) vs. Time for Individual Patients at Site of Compression

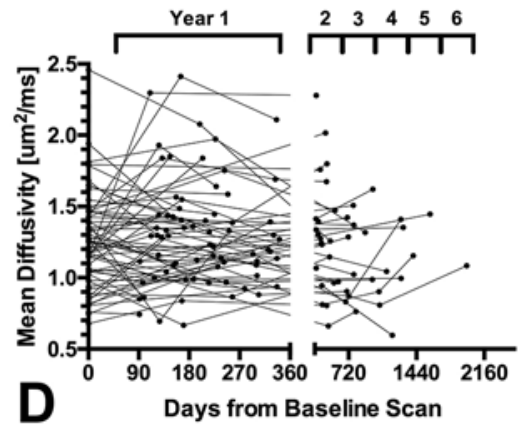

Percentage Change in Mean Diffusivity (MD) vs. Time for Individual Patients at Site of Compression

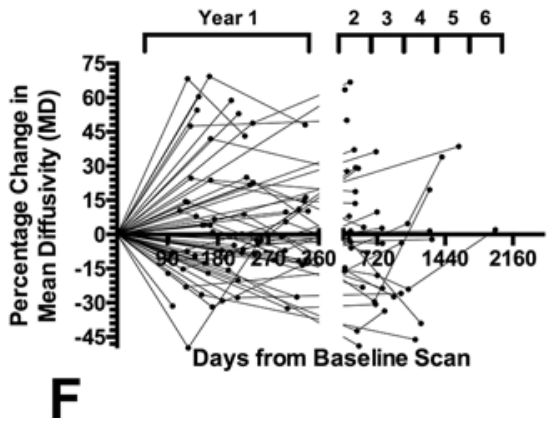

FIG. 3. Graphs showing long-term temporal DTI measurements and trends in patients with cervical spondylosis. A: The FA evaluated over time at the site of compression (solid circles) and in C1-2 reference tissue (open circles). B: The MD evaluated over time at the site of compression (solid circles) and in C1-2 reference tissue (open circles). C: The FA values at the site of compression for individual patients with cervical spondylosis (connected by lines). D: The MD values at the site of compression for individual patients with cervical spondylosis (connected by lines). E: Percentage change in FA measurements at the site of compression over the surveillance period, with respect to the first imaging examination time point. F: Percentage change in MD measurements at the site of compression over the surveillance period, with respect to the first imaging examination time point.

of multiple DTI measurements of MD and FA of approximately $8.7 \%$ and $4.6 \%$, respectively, ${ }^{12}$ within reference tissues in 15 patients evaluated over approximately 200 days, which is slightly lower than the $10.7 \%$ and $6.2 \%$ COVs reported in the current study.

The average FA and MD measurements reported in reference $\mathrm{C} 1-2$ tissues within the current study are consistent with those previously reported in the normal cervical spinal cord. For example, we report average FA and MD measurements of 0.61 and $0.91 \mu \mathrm{m}^{2} / \mathrm{msec}$, respectively, whereas
Mamata et al. ${ }^{28}$ reported values of 0.70 and $0.81 \mu \mathrm{m}^{2} / \mathrm{msec}$, Facon et al. ${ }^{14}$ reported values of approximately 0.75 and $1.0 \mu \mathrm{m}^{2} / \mathrm{msec}$, and Budzik et al. ${ }^{6}$ reported values of 0.54 and $0.78 \mathrm{um}^{2} / \mathrm{msec}$. Additionally, Jones et al. ${ }^{23}$ reported FA values of the spinal cord at C2-3 of approximately 0.64 , which is also consistent with our reported values at $\mathrm{C} 1-2$.

In general, we observed an increased MD and decreased FA at the site of compression compared with reference (normal) values. This is consistent with previous observations in patients with cervical spondylosis. ${ }^{6,10,12,14 \text {, }}$ 


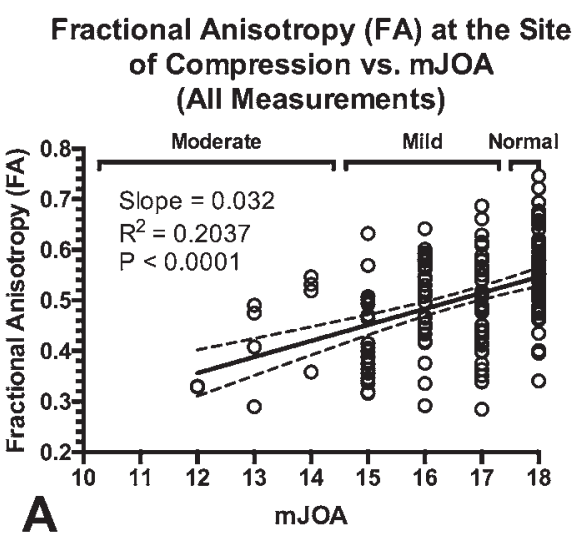

Mean Diffusivity (MD) at the Site of Compression vs. mJOA

(All Measurements)

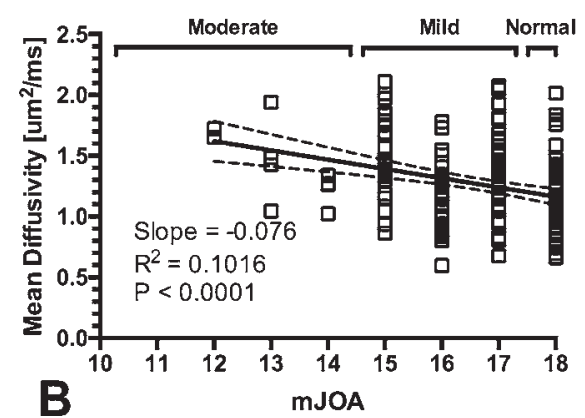

Fractional Anisotropy (FA) at the Site of Compression vs. mJOA (Per Patient)

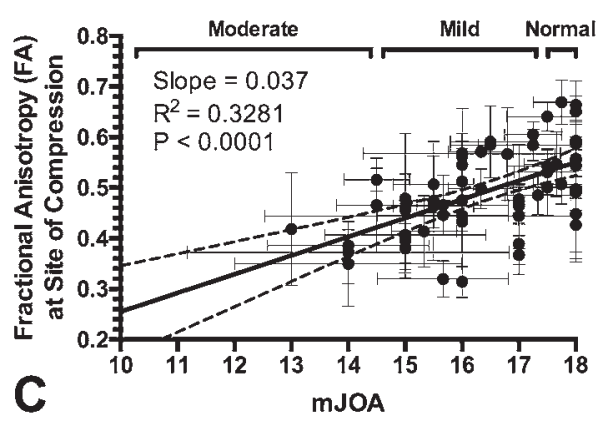

Mean Diffusivity (MD) at the Site of Compression vs. mJOA (Per Patient)

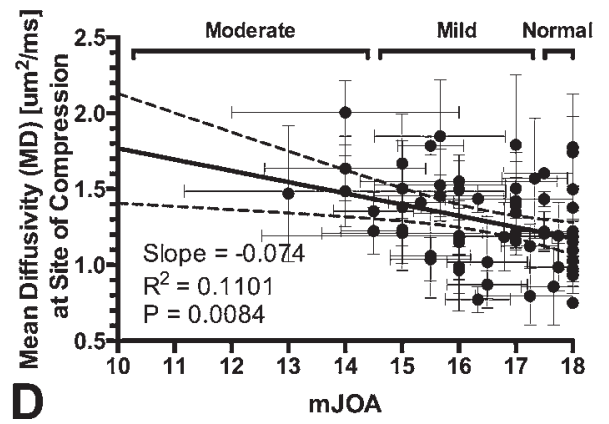

FIG. 4. Graphs showing correlation between DTI measurements of the spinal cord at the site of compression and functional impairment assessed with the mJOA scale. A: The FA measurements at the site of compression versus mJOA score determined using all available measurements from all patients $\left(R^{2}=0.2037, p<0.0001\right)$. B: The MD measurements at the site of compression versus mJOA score determined using all available measurements $\left(R^{2}=0.1016, p<0.0001\right)$. C: Association between FA measurements at the site of compression and mJOA score evaluated per patient, averaging multiple DTI and neurological assessment examinations $\left(R^{2}=0.3281, p<0.0001\right)$. $D$ : Association between MD at the site of compression and mJOA score evaluated per patient by averaging multiple DTI and neurological examinations $\left(R^{2}=0.1101, p=0.0084\right)$.

22,23,28 Additionally, we observed a significant correlation between degree of neurological impairment assessed using the mJOA score and DTI measurements, including FA and MD, which is also consistent with previous clinical studies. ${ }^{13,23}$ Together, these results support the hypothesis that DTI measurements at the site of compression may be valuable for estimating the degree of neurological impairment in patients with cervical spondylosis.

The present investigation is novel and clinically significant for several reasons. First, having performed 183 separate MRI scans in a cohort of 66 patients, this is the first and largest study in which the temporal stability and reproducibility of DTI were evaluated in patients with advanced cervical spondylosis. This helps to address a gap in our knowledge base regarding the stability and efficacy of repeated DTI measurements over time; the vast majority of studies have been focused on single time-point assessments. Second, we were able to determine the expected change in mJOA score based on changes in FA and MD measurements. Fractional anisotropy was shown to decrease by approximately 0.032 units and MD was demonstrated to increase approximately $0.084 \mu \mathrm{m}^{2} / \mathrm{msec}$ for every point decrease in $\mathrm{mJOA}$ score. Last, when examining each individual patient by averaging their DTI and mJOA findings over their respective surveillance period, FA and MD were strongly correlated with sensitivity to their mJOA score. Taken together, these 3 key findings suggest that DTI could potentially be used as a method to longitudinally assay spinal cord integrity in patients with asymptomatic cervical stenosis and CSM who are being treated nonoperatively. Additional future study with a larger cohort of patients and longer follow-up time will be needed.

\section{Study Limitations}

Although DTI can provide new information regarding the health of the spinal cord, there remain limitations to widespread use due to technical challenges and issues with consistency in slice prescriptions between evaluation time points. Many of the technical challenges relate to the small size and movement of the cord-namely, inaccuracies arising from partial volume and susceptibility-related artifacts. Additionally, general issues with consistency and accuracy of slice prescription for even the anatomical evaluation of stenosis are well documented, ${ }^{18,40}$ and thus adequate attention should be given to alignment of slices across examinations in the same patient. 
It is also important to note that technological advances in MR system hardware and software evolved over the surveillance period in the current study, resulting in slight variability in the acquisition protocols over time. Thus, our measurements of variability and temporal stability documented in the present study reflect the realistic variations in DTI measurements expected during routine clinical use of this modality for evaluation of the spinal cord by using clinically available, state-of-the-art acquisition protocols. The current version of our acquisition protocol that we used for the last 3 years of examinations, consisting of 30 diffusion-sensitizing directions and 4 averages for $\mathrm{b}=0$ seconds $/ \mathrm{mm}^{2}$ images, has tolerable accuracy in DTI parameter estimation ${ }^{7,43}$ and is consistent with current consensus recommendations from the scientific community. ${ }^{39}$

\section{Conclusions}

Quantitative DTI measurements of the spinal cord in patients with advanced cervical spondylosis have a median COV of $5 \%-10 \%$, which is similar to anatomical measurements at the site of compression. The mean measurements of FA and MD in normal reference tissues, as well as the increase in MD and decrease in FA in patients with increasing neurological impairment, are consistent with those in previous studies. Results also suggest that FA within the spinal cord is slightly more sensitive to neurological function and more stable than measures of MD. The reproducibility of these measurements and significant correlation with functional outcome status suggest a potential role in the evaluation and longitudinal surveillance of nonoperatively treated patients.

\section{Acknowledgments}

The NIH gave clinical or research support for the study described (includes equipment or material).

\section{References}

1. Arnold JG Jr: The clinical manifestations of spondylochondrosis (spondylosis) of the cervical spine. Ann Surg 141:872-889, 1955

2. Bailey P, Casa Major L: Osteoarthritis of the spine as a cause of compression of the spinal cord and its roots. J Nerv Ment Dis 38:588-609, 1911

3. Baptiste DC, Fehlings MG: Pathophysiology of cervical myelopathy. Spine J 6 (6 Suppl):190S-197S, 2006

4. Boden SD, McCowin PR, Davis DO, Dina TS, Mark AS, Wiesel S: Abnormal magnetic-resonance scans of the cervical spine in asymptomatic subjects. A prospective investigation. J Bone Joint Surg Am 72:1178-1184, 1990

5. Brain WR, Northfield D, Wilkinson M: The neurological manifestations of cervical spondylosis. Brain 75:187-225, 1952

6. Budzik JF, Balbi V, Le Thuc V, Duhamel A, Assaker R, Cotten A: Diffusion tensor imaging and fibre tracking in cervical spondylotic myelopathy. Eur Radiol 21:426-433, 2011

7. By S, Smith AK, Dethrage LM, Lyttle BD, Landman BA, Creasy JL, et al: Quantifying the impact of underlying measurement error on cervical spinal cord diffusion tensor imaging at 3T. J Magn Reson Imaging 44:1608-1618, 2016

8. Clarke E, Robinson PK: Cervical myelopathy: a complication of cervical spondylosis. Brain 79:483-510, 1956

9. Cooke FJ, Blamire AM, Manners DN, Styles P, Rajagopalan
B: Quantitative proton magnetic resonance spectroscopy of the cervical spinal cord. Magn Reson Med 51:1122-1128, 2004

10. Demir A, Ries M, Moonen CT, Vital JM, Dehais J, Arne P, et al: Diffusion-weighted MR imaging with apparent diffusion coefficient and apparent diffusion tensor maps in cervical spondylotic myelopathy. Radiology 229:37-43, 2003

11. Ellenberg MR, Honet JC, Treanor WJ: Cervical radiculopathy. Arch Phys Med Rehabil 75:342-352, 1994

12. Ellingson BM, Salamon N, Grinstead JW, Holly LT: Diffusion tensor imaging predicts functional impairment in mild-to-moderate cervical spondylotic myelopathy. Spine J 14:2589-2597, 2014

13. Ellingson BM, Salamon N, Hardy AJ, Holly LT: Prediction of neurological impairment in cervical spondylotic myelopathy using a combination of diffusion MRI and proton MR spectroscopy. PLoS One 10:e0139451, 2015

14. Facon D, Ozanne A, Fillard P, Lepeintre JF, Tournoux-Facon C, Ducreux D: MR diffusion tensor imaging and fiber tracking in spinal cord compression. AJNR Am J Neuroradiol 26:1587-1594, 2005

15. Fehlings MG, Skaf G: A review of the pathophysiology of cervical spondylotic myelopathy with insights for potential novel mechanisms drawn from traumatic spinal cord injury. Spine (Phila Pa 1976) 23:2730-2737, 1998

16. Fernández de Rota JJ, Meschian S, Fernández de Rota A, Urbano V, Baron M: Cervical spondylotic myelopathy due to chronic compression: the role of signal intensity changes in magnetic resonance images. J Neurosurg Spine 6:17-22, 2007

17. Friedenberg ZB, Miller WT: Degenerative disc disease of the cervical spine. J Bone Joint Surg Am 45:1171-1178, 1963

18. Henderson L, Kulik G, Richarme D, Theumann N, Schizas C: Is spinal stenosis assessment dependent on slice orientation? A magnetic resonance imaging study. Eur Spine J 21 (Suppl 6):S760-S764, 2012

19. Holly LT, Ellingson BM, Salamon N: Metabolic imaging using proton magnetic spectroscopy as a predictor of outcome following surgery for cervical spondylotic myelopathy. J Spinal Disord Tech 30:E615-E619, 2017

20. Holly LT, Freitas B, McArthur DL, Salamon N: Proton magnetic resonance spectroscopy to evaluate spinal cord axonal injury in cervical spondylotic myelopathy. J Neurosurg Spine 10:194-200, 2009

21. Hori M, Fukunaga I, Masutani Y, Nakanishi A, Shimoji K, Kamagata K, et al: New diffusion metrics for spondylotic myelopathy at an early clinical stage. Eur Radiol 22:17971802,2012

22. Hori M, Okubo T, Aoki S, Kumagai H, Araki T: Line scan diffusion tensor MRI at low magnetic field strength: feasibility study of cervical spondylotic myelopathy in an early clinical stage. J Magn Reson Imaging 23:183-188, 2006

23. Jones JG, Cen SY, Lebel RM, Hsieh PC, Law M: Diffusion tensor imaging correlates with the clinical assessment of disease severity in cervical spondylotic myelopathy and predicts outcome following surgery. AJNR Am J Neuroradiol 34:471-478, 2013

24. Kara B, Celik A, Karadereler S, Ulusoy L, Ganiyusufoglu K, Onat L, et al: The role of DTI in early detection of cervical spondylotic myelopathy: a preliminary study with 3-T MRI. Neuroradiology 53:609-616, 2011

25. Karadimas SK, Erwin WM, Ely CG, Dettori JR, Fehlings MG: Pathophysiology and natural history of cervical spondylotic myelopathy. Spine (Phila Pa 1976) 38 (22 Suppl 1):S21-S36, 2013

26. LaRocca H: Cervical spondylotic myelopathy: natural history. Spine (Phila Pa 1976) 13:854-855, 1988

27. Lees F, Turner JW: Natural history and prognosis of cervical spondylosis. BMJ 2:1607-1610, 1963 
28. Mamata H, Jolesz FA, Maier SE: Apparent diffusion coefficient and fractional anisotropy in spinal cord: age and cervical spondylosis-related changes. J Magn Reson Imaging 22:38-43, 2005

29. Mastronardi L, Elsawaf A, Roperto R, Bozzao A, Caroli M, Ferrante M, et al: Prognostic relevance of the postoperative evolution of intramedullary spinal cord changes in signal intensity on magnetic resonance imaging after anterior decompression for cervical spondylotic myelopathy. J Neurosurg Spine 7:615-622, 2007

30. Matsuda Y, Miyazaki K, Tada K, Yasuda A, Nakayama T, Murakami H, et al: Increased MR signal intensity due to cervical myelopathy. Analysis of 29 surgical cases. J Neurosurg 74:887-892, 1991

31. Matsumoto M, Toyama Y, Ishikawa M, Chiba K, Suzuki N, Fujimura Y: Increased signal intensity of the spinal cord on magnetic resonance images in cervical compressive myelopathy. Does it predict the outcome of conservative treatment? Spine (Phila Pa 1976) 25:677-682, 2000

32. McCormick WE, Steinmetz MP, Benzel EC: Cervical spondylotic myelopathy: make the difficult diagnosis, then refer for surgery. Cleve Clin J Med 70:899-904, 2003

33. Mehalic TF, Pezzuti RT, Applebaum BI: Magnetic resonance imaging and cervical spondylotic myelopathy. Neurosurgery 26:217-227, 1990

34. Morio Y, Yamamoto K, Kuranobu K, Murata M, Tuda K: Does increased signal intensity of the spinal cord on MR images due to cervical myelopathy predict prognosis? Arch Orthop Trauma Surg 113:254-259, 1994

35. Nurick $S$ : The natural history and the results of surgical treatment of the spinal cord disorder associated with cervical spondylosis. Brain 95:101-108, 1972

36. Pavlov H, Torg JS, Robie B, Jahre C: Cervical spinal stenosis: determination with vertebral body ratio method. Radiology 164:771-775, 1987

37. Puzzilli F, Mastronardi L, Ruggeri A, Lunardi P: Intramedullary increased MR signal intensity and its relation to clinical features in cervical myelopathy. J Neurosurg Sci 43:135139,1999

38. Salamon N, Ellingson BM, Nagarajan R, Gebara N, Thomas A, Holly LT: Proton magnetic resonance spectroscopy of human cervical spondylosis at 3T. Spinal Cord 51:558-563, 2013

39. Samson RS, Lévy S, Schneider T, Smith AK, Smith SA, Cohen-Adad J, et al: ZOOM or Non-ZOOM? Assessing spinal cord diffusion tensor imaging protocols for multi-centre studies. PLoS One 11:e0155557, 2016

40. Schönström N: The significance of oblique cuts on CT scans of the spinal canal in terms of anatomic measurements. Spine (Phila Pa 1976) 13:435-436, 1988

41. Schwartz ED, Cooper ET, Fan Y, Jawad AF, Chin CL, Nis- sanov J, et al: MRI diffusion coefficients in spinal cord correlate with axon morphometry. Neuroreport 16:73-76, 2005

42. Suk KS, Kim KT, Lee JH, Lee SH, Kim JS, Kim JY: Reevaluation of the Pavlov ratio in patients with cervical myelopathy. Clin Orthop Surg 1:6-10, 2009

43. Tu TW, Kim JH, Wang J, Song SK: Full tensor diffusion imaging is not required to assess the white-matter integrity in mouse contusion spinal cord injury. J Neurotrauma 27:253262, 2010

44. Wen CY, Cui JL, Liu HS, Mak KC, Cheung WY, Luk KD, et al: Is diffusion anisotropy a biomarker for disease severity and surgical prognosis of cervical spondylotic myelopathy? Radiology 270:197-204, 2014

45. Wen CY, Cui JL, Mak KC, Luk KD, Hu Y: Diffusion tensor imaging of somatosensory tract in cervical spondylotic myelopathy and its link with electrophysiological evaluation. Spine J 14: 1493-1500, 2014

46. Yonenobu K, Abumi K, Nagata K, Taketomi E, Ueyama K: Interobserver and intraobserver reliability of the Japanese Orthopaedic Association scoring system for evaluation of cervical compression myelopathy. Spine (Phila Pa 1976) 26:1890-1895, 2001

47. Young WF: Cervical spondylotic myelopathy: a common cause of spinal cord dysfunction in older persons. Am Fam Physician 62:1064-1070, 1073, 2000

48. Yue WM, Tan SB, Tan MH, Koh DC, Tan CT: The Torg--Pavlov ratio in cervical spondylotic myelopathy: a comparative study between patients with cervical spondylotic myelopathy and a nonspondylotic, nonmyelopathic population. Spine (Phila Pa 1976) 26:1760-1764, 2001

\section{Disclosures}

Dr. Holly is a consultant for and receives royalties from Medtronic.

\section{Author Contributions}

Conception and design: Ellingson, Salamon, Holly. Acquisition of data: Ellingson, Woodworth. Analysis and interpretation of data: all authors. Drafting the article: Ellingson, Salamon, Holly. Critically revising the article: Ellingson, Salamon, Holly. Reviewed submitted version of manuscript: all authors. Approved the final version of the manuscript on behalf of all authors: Ellingson. Statistical analysis: Ellingson. Administrative/technical/material support: Holly. Study supervision: Holly.

\section{Correspondence}

Benjamin M. Ellingson: University of California, Los Angeles, CA. bellingson@mednet.ucla.edu. 